\title{
Etiology and Management of Small Bowel Obstruction with Special Reference to Role of Laparoscopy-A Single Centre Experience
}

\author{
Showkat Ahmad Mir1, Rauf Ahmad Wani', Yaqoob Hassan²*, Fazl Q. Parray², \\ Nisar Ahmad Chowdri ${ }^{1}$ \\ ${ }^{1}$ Department of General and Minimal Access Surgery, SKIMS, Srinagar, India \\ ${ }^{2}$ Department of General and Minimal Access Surgery, Sher-i-Kashmir Institute of Medical Sciences, Medical College, Srinagar, India \\ Email: abuabdulmuizz786@gmail.com,roufw64@hotmail.com, ^dryaqoobwani@gmail.com
}

How to cite this paper: Mir, S.A., Wani, R.A., Hassan, Y., Parray, F.Q. and Chowdri, N.A. (2019) Etiology and Management of Small Bowel Obstruction with Special Reference to Role of Laparoscopy-A Single Centre Experience. Surgical Science, 10, 113-126.

https://doi.org/10.4236/ss.2019.104015

Received: February 26, 2019

Accepted: April 7, 2019

Published: April 10, 2019

Copyright $\odot 2019$ by author(s) and Scientific Research Publishing Inc. This work is licensed under the Creative Commons Attribution-NonCommercial International License (CC BY-NC 4.0). http://creativecommons.org/licenses/by-nc/4.0/

\begin{abstract}
Background: Small bowel obstruction ( $\mathrm{SBO}$ ) is the commonly diagnosed disease in the emergency department. The diagnosis and management of small bowel obstruction varies among institutions. The role of laparoscopy in the management of small bowel obstruction is debatable. Aim: To study the profile, clinical features and management options of patients of small bowel obstruction with special emphasis on role of laparoscopy. Material and Methods: 51 consecutive patients with documented small bowel obstruction admitted in our department were studied from June 2016 to December 2018. All the patients above 12 years of age with small bowel obstruction were included. The diagnosis of small bowel obstruction (SBO) was made in these cases by detailed history, clinical examination and investigations. All these patients were received and adequately resuscitated. Non-operative (conservative) treatment was confirmed to those who met the standard parameters for such treatment and early surgical treatment was carried out whenever indicated. The operative procedure done was laparoscopy or open depending on the clinical features and condition of patient. Results: In our study, $51 \mathrm{pa}-$ tients mean age was 44.7 years with 27 Males and 24 females. 28 (54.9\%) patients of our study presented with continuous abdominal pain followed by colicky pain in 23 (45.1\%) patients. 34 (66.7\%) patients on admission gave history of effortless vomiting. Distension of abdomen was present in 30 (58.8\%) patients and constipation was present in 40 (78.4\%) of our studied patients. On CT scan interloop fluid was present in $23(45.1 \%)$ patients while transition point was present in $31(60.8 \%)$ patients. Adhesion obstruction was found to be most common cause of obstruction 27 (52.9\%) patients, followed by malignancy in 8 (15.7). 25 (49.0\%) patients were managed conservatively and $26(51 \%)$ were operated. 11 (21.6\%) patients
\end{abstract}


underwent laparoscopic adhesiolysis and 15 (29.4\%) underwent exploratory laparotomy. The average operative time, recovery of bowel moments, hospital stay, and post-operative complications were less in the laparoscopic group than open group with statically significant difference between the two. Conclusion: Post-operative adhesion obstruction is the most common cause of small bowel obstruction. Laparoscopy is a safe and effective tool for the management of acute intestinal obstruction provided it is performed in selected patients by skilled surgeon.

\section{Keywords}

Bowel Obstruction, Laparoscopy, Adhesions, Non-Operative, Small Bowel

\section{Introduction}

Small bowel obstruction is defined as a partial or complete interference with the passage of stool distally in the small intestine [1]. Small bowel obstruction remains an important morbidity, accounting for up to $15 \%$ of surgical admissions for acute non-traumatic abdomen pain [2]. The small bowel is involved in $60 \%$ to $80 \%$ cases of intestinal obstruction [3]. In spite of advances in imaging and better understanding of pathophysiology of small bowel, its obstruction is still frequently misdiagnosed [3]. Despite advances in the treatment of this condition, the attendant mortality is still high and remains in range of 5\% to $11 \%$ [4].

Small bowel obstruction is mainly of two types [5], mechanical or dynamic and functional or adynamic. The latter form of obstruction is referred as ileus or pseudo obstruction [6]. Mechanical small bowel obstruction is the most frequently encountered surgical disorder of the small intestine. Although a wide range of aetiologies for this condition exist, intra-abdominal adhesions related to prior abdominal and pelvic surgeries is the etiologic factor in up to $70 \%$ of cases small bowel obstruction in developed countries [2]. Less prevalent aetiologies for small bowel obstruction includes Crohn's disease, volvulus, Intussusception, radiation-induced stricture, post ischemic stricture, foreign body, gall stone ileus, diverticulitis, Meckel's diverticulum, congenital abnormalities (e.g. webs, duplications and malrotation) and small bowel neoplasms. Fewer than $3 \%$ of cases are caused by primary small intestinal neoplasms [5]. Other common causes of small bowel obstruction in the developing world are abdominal tuberculosis [7] and worm obstruction [8].

The cardinal symptoms that characterize mechanical small bowel obstruction include colicky abdominal pain, vomiting, abdominal distension and obstipation (failure of passage of flatus and faeces) [2] [3] [4] [5] [6]. Initially pain tends to be diffuse, poorly localized, episodic and crampy in nature. However with the onset of bowel ischemia pain becomes constant. Continued passage of flatus and stool beyond 6 to 12 hours after onset of symptoms are characteristic of partial rather than complete obstruction. Vomiting occurs early in proximal small bowel obstruction but may be absent or develop late in distal obstruction. Char- 
acter of vomitus also differs as far as site of obstruction is concerned. Visible peristalsis may be seen in patients while in others distension may be prominent which is usually centrally placed. Abnormal auscultation reveals hyperactive bowel sounds. Laboratory findings reflect intravascular volume depletion, hemoconcentration and electrolyte imbalance. Features of strangulated obstruction include tachycardia, localized abdominal-tenderness, fever, marked leukocytosis and acidosis [9].

In addition to very careful and thorough history and physical examination, all patients require base line laboratory tests including complete blood counts serum electrolytes and $x$-ray abdomen standing and supine. Plane radiograph of abdomen is an important diagnostic tool. Radiographs have accuracy of $67 \%$ $83 \%$ in the diagnosis of small bowel obstruction, with reported sensitivity of $64 \%$ - $68 \%$ and specificity of $79 \%$ - 83\% [2]. The cardinal findings include dilated loops of small intestine on the supine radiographs and multiple air fluid levels which are in step ladder pattern on erect radiograph. In general dilated loops of small intestine are defined as those larger than $3 \mathrm{~cm}$ in diameter [6].

Fluoroscopy and follow-through examination with oral contrast agents have a limited role in the diagnosis of small bowel obstruction but may be useful in determining the severity of obstruction [2].

Computed tomography scanning is $80 \%$ - $90 \%$ sensitive and $70 \%-90 \%$ specific in the detection of small bowel obstruction [10]. The findings of small bowel obstruction include a discrete transition zone with dilatation of bowel proximally, decompression of bowel distally, intraluminal contrast that does not pass beyond the transition zone, and a colon containing little gas or fluid. Other diagnostic tests used are CT enteroclysis, USG abdomen and water soluble contrast medium study.

Initial treatment consists of aggressive fluid resuscitation, bowel decompression, administration of analgesia and antiemetics. Almost all patients benefit from an initial 12 - 14 hours of resuscitation and decompression in case of complete obstruction; resuscitation and decompression can usually be extended for a longer period of time in those patients with partial obstruction who exhibit no signs of progression. Because of the inability to detect reversible ischemia, there is a substantial risk of progression to irreversible ischemia when surgery is delayed for an extended period of time, especially in suspected complete obstruction [11].

The administration of hypertonic water-soluble contrast agents, such as gastrograffin used in upper GI and small-bowel follow-through examinations, causes a shift of fluid into the intestinal lumen, thereby increasing the pressure gradient across the site of obstruction. This effect may accelerate resolution of partial small bowel obstruction, however, whether administration of water-soluble contrast agents increases the probability that an episode of bowel obstruction can be successfully managed non-operatively remains controversial and requires further study. 
A strangulated obstruction is a surgical emergency. In patients with a complete small bowel obstruction, the risk of strangulation is high and early surgical intervention is warranted. Patients with simple complete obstruction in whom non-operative trails fail, also need surgical treatment.

The most important factor predicting the recurrence of small bowel obstruction is the number of prior episodes. Non-operative management appears reasonable for stable patients who are having their first episode. Operative strategies appear best for those experiencing second episode. Neither strategy yields acceptable out comes in patients experiencing third or later episodes [12].

Post-operative adhesions are the most frequent cause of small bowel obstruction. Even after operative intervention for small bowel obstruction recurrences are common. Laparoscopy is believed to reduce the risk of adhesions compared to laparotomy. Laparoscopy has revolutionized the elective management of many surgical conditions and results in reduced morbidity and shorter hospital stays. Advances in technology and increased experience have made laparoscopic treatment of acute small bowel obstruction possible. However, laparotomy remains the standard surgical approach to acute small bowel obstruction. Small bowel obstruction has been seen as a relative contraindication for minimal access surgery. This is due to the belief that in the presence of distended bowel, which reduces visibility and increases the risk of bowel injury, the conversion rates and morbidity would be unacceptable [13].

Laparoscopy is known to cause fewer intra-abdominal adhesions than open surgery. Laparoscopic treatment of acute small bowel obstruction was first described by Bastug et al. [14] and has since been reported by others, but studies comparing the result of laparoscopic and conventional treatment of this condition are lacking. The conversion rate of $51.9 \%$ in the study was slightly higher than previously values of about $45 \%$ [15]. The main reason for conversion was an obscured view due to intestinal distension in combination with extensive adhesions. A reduced field of vision together with the vulnerability of the bowel limits the use of laparoscopy and may explain why laparoscopy for acute small bowel obstruction has the highest rate of conversion. There is evidence that laparoscopic treatment of acute small bowel obstruction leads to a higher rate of bowel injury than conventional surgery.

Adhesions from prior surgery are the most common cause of small bowel obstruction. The incidence of an adhesive small bowel obstruction after open abdominal surgery is between $12 \%$ and $17 \%$. Historically, laparotomy and open adhesiolysis have been the treatment for patients requiring surgery for small bowel obstruction. Unfortunately, this often leads to further formation of intra-abdominal adhesions with approximately $10 \%-30 \%$ of patients requiring another laparotomy for recurrent bowel obstruction [14].

Laparoscopic adhesiolysis for small bowel obstruction has a number of potential advantages like lesser postoperative pain, quicker return of intestinal function, shorter hospital stay, reduced recovery time, earlier return to full activity, 
fewer wound complications and decreased postoperative adhesion formation. Although laparoscopic adhesiolysis have many potential advantages, the most important aspect to a successful outcome is proper patient's selection and surgical judgement. When reviewing the literature, there are no clear guidelines that state which patients are best for laparoscopic adhesiolysis, however, there are several factors that have been shown to predict a successful outcome.

Our hospital is a high volume centre. No such study has been undertaken in our population, so we have undertaken this study to look at the feasibility and safety of laparoscopy in such cases in our hospital.

\subsection{Aims and Objectives}

1) To study patient profile and clinical features and management options of patients of small bowel obstruction.

2) To study role and feasibility of laparoscopy in small bowel obstruction.

\subsection{Material and Methods}

Study Design: Prospective Observational Study.

Study Area: The study was conducted in the department of General and Minimal Access Surgery, Sher-i-kashmir Institute of Medical Sciences, Srinagar.

Study Population: Patients above 12 years of age belonging to either of the sex with documented small bowel obstruction were taken up for the study.

Study Sample: Our study consisted of 51 consecutive patients with documented small bowel obstruction admitted in our department.

Study Duration: The study was conducted from June 2016 to December 2018.

Inclusion Criteria: All patients above 12 years of age with small bowel obstruction.

Exclusion Criteria: All patients below 12 years of age with small bowel obstruction.

\subsection{Methodology}

The diagnosis of small bowel obstruction (SBO) was made in our study by detailed history, clinical examination and investigations. All the patients were subjected to base line investigations which included complete haemogram, urine analysis, blood urea, serum creatinine, liver function test, serum electrolytes, Electrocardiography, X-ray chest, X-ray abdomen and Ultrasonography. All the patients were subjected to Contrast Enhanced Computed Tomography (CECT) of abdomen.

All these patients were received and resuscitated by conventional methods of intravenous fluids, nasogastric suction and broad spectrum antibiotics while preparing patients for surgery. A special emphasis was laid on ensuring good functions of kidney by monitoring adequate urine output.

The management of patients as individualized, non-operative (conservative) treatment was confirmed to those who met the standard parameters for such 
treatment and early surgical treatment was carried out whenever indicated. The operative procedure done was laparoscopy or open depending on the clinical features and condition of patient. There was no hesitation for conversion (laparoscopic to open) if needed.

On satisfactory response to conservative treatment, patients were allowed oral feeds only when intestines start functioning (i.e. appearance of bowel sounds and passing of flatus/stools). On complete resolution of symptoms, patients were discharged and attached to Out Patient Department (OPD) fallow up.

Patients, who were subjected to surgery, were strictly monitored in post-operative period. Postoperative antibiotics were used in all cases. All the patients were fallowed weekly for first month followed by monthly basis for next 6 months on outpatient basis.

\section{Statistical Methods}

The recorded data was compiled and entered in a spreadsheet (Microsoft Excel) and then exported to data editor of SPSS Version 20.0 (SPSS Inc., Chicago, Illinois, USA). Continuous variables were expressed as Mean \pm SD and categorical variables were summarized as frequencies and percentages. Graphically the data was presented by bar diagrams. Student's independent t-test was employed for comparing continuous variables. Chi-square test or Fisher's exact test, whichever appropriate, was applied for comparing categorical variables. A P-value of less than 0.05 was considered statistically significant. All P-values were two tailed.

\section{Results}

In our study of 51 patients, $2(3.9 \%)$ patients were <20 years of age, $12(23.5 \%)$ patients aged 20 - 34 years, $16(31.4 \%)$ patients were $35-49$ years, and 14 (27.5\%) patients were 50 - 64 years. There were 7 (13.7\%) patients who aged $\geq$ 65 years. The mean age in our study population was 44.7 years. Males were little more in our study than females, 27 (52.9\%) vs. 24 (47.1\%).

28 (54.9\%) patients of our study presented with continuous abdominal pain followed by colicky pain in $23(45.1 \%)$ patients. $34(66.7 \%)$ patients on admission gave history of effortless vomiting while as $6(11.8 \%)$ patients had projectile and 6 (11.8) patients gave history of sudden vomiting. 5 (9.8\%) patients had no history of vomiting at all. Distension of abdomen was present in $30(58.8 \%)$ patients and constipation was present in $40(78.4 \%)$ of our studied patients.

On CT scan interloop fluid was present in $23(45.1 \%)$ patients while transition point was present in $31(60.8 \%)$ patients. $16(31.4 \%)$ patients had $3-4 \mathrm{~cm}$ diameter of bowl on CECT findings (Table 1).

Adhesion obstruction was found to be most common cause of obstruction, and was in 27 (52.9\%) patients fallowed by malignancy in 8 (15.7\%) as shown in Table 2.

Against a total of 51 patients, adhesion obstruction was seen in 27 patients of which $13(48.14 \%)$ patients underwent surgery, $3(60 \%)$ of 5 patients with fe- 
caloma were also operated. In 8 malignancy patients, 5 (62.5\%) underwent surgery leaving 3 patients who had diffuse peritoneal metastasis and could not be operated upon. Two (100\%) patients with phytobezarand 2 (66\%) out of 3 patients with tuberculosis underwent surgery. Patients with crohn's disease and worm obstruction were managed conservatively. There was only one patient with Para duodenal hernia and had to underwent surgery (Figure 1).

Regarding management of our cases of small bowel obstruction, out of 51 patients 25 (49.0\%) were managed conservatively and 26 (51\%) were operated. Patients who were managed conservatively were closely monitored for any signs of strangulation like tachycardia, local abdominal tenderness, fever, leucocytosis and acidosis. $11(21.6 \%)$ patients underwent laparoscopic adhesiolysis and 15 (29.4\%) underwent exploratory laparotomy.

An average operative time for laparoscopic adhesiolysis was 84.5 minutes and explorative laparotomy was 134.3 minutes as shown in Table 3. The difference between the two groups was statistically significant with a P-value of $<0.05$.

Patients who underwent laparoscopic adhesiolysis had mean hospital stay of 7.8 days while as patients who had undergone explorative laparotomy were discharged after 15 days as shown in Table 4.

Mean time (days) for return of bowel sounds was $1.3 \pm 0.504$ days in laparoscopic adhesiolysis and $2.1 \pm 0.743$ days in exploratory laparotomy as shown in Table 5.

Mean time (days) for passage of flatus/stools was $2.5 \pm 0.786$ days in laparoscopic adhesiolysiss and $3.6 \pm 0.899$ days in exploratory laparotomy as shown in Table 6.

Postoperative obstruction was seen in 1 (9.1\%) patients of laparoscopic adhesiolysis while as $3(20 \%)$ patients suffered postoperative obstruction in exploratory laparotomy group. Anastomotic leakage was seen again in $3(20.0 \%)$ patients of exploratory laparotomy, small bowel tear and postoperative ileus was seen in $1(9.1 \%)$ patients each in laparoscopic group. Wound infection was seen in $4(26.7 \%)$ in exploratory laparotomy group as shown in Table 7.

There was no mortality in laparoscopic adhesiolysis group while as only 1 (6.7\%) patient in exploratory laparotomy group due to post-operative sepsis.

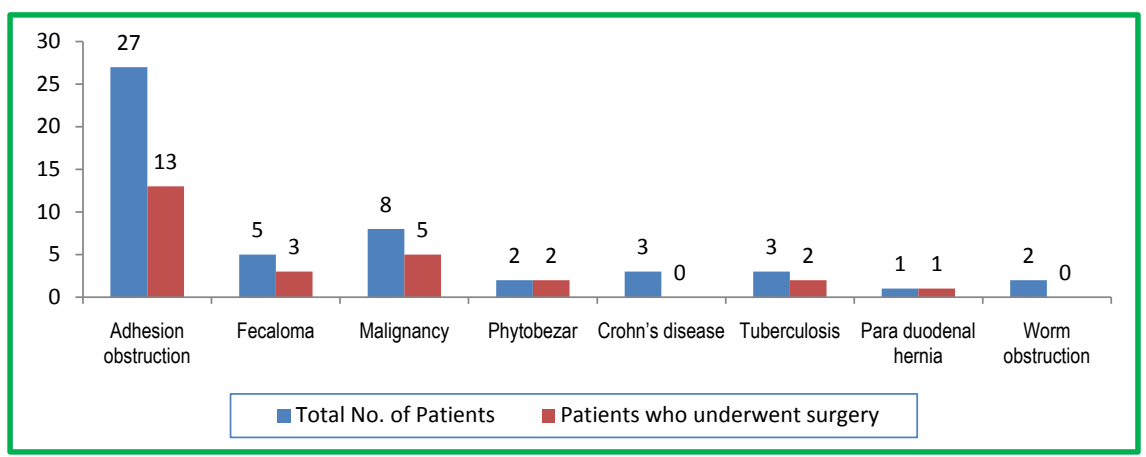

Figure 1. Surgery according to etiology. 
Table 1. CT findings in study patients.

\begin{tabular}{cccc}
\hline CT Findings & & Frequency & Percentage \\
\hline \multirow{2}{*}{ Interloop Fluid } & Present & 23 & 45.1 \\
& Absent & 28 & 54.9 \\
\multirow{3}{*}{ Transition Point } & Present & 31 & 60.8 \\
& Absent & 20 & 39.2 \\
\multirow{3}{*}{ Diameter of Bowl (cm) } & $2-3$ & 13 & 25.5 \\
& $3-4$ & 16 & 31.4 \\
\hline
\end{tabular}

Table 2. Etiology of study patients.

\begin{tabular}{ccc}
\hline Etiology & Frequency & Percentage \\
\hline Adhesion obstruction & 27 & 52.9 \\
Fecaloma & 5 & 9.8 \\
Malignancy & 8 & 15.7 \\
Phytobezar & 2 & 3.9 \\
Crohn's disease & 3 & 5.9 \\
Tuberculosis & 3 & 5.9 \\
Para duodenal hernia & 1 & 2.0 \\
Worm obstruction & 2 & 3.9 \\
Total & 51 & 100 \\
\hline
\end{tabular}

Table 3. Operative time (minutes) as per management in study patients.

\begin{tabular}{cccccc}
\hline $\begin{array}{c}\text { Operative time } \\
\text { (Minutes) }\end{array}$ & N & Mean & SD & Range & P-value \\
\hline LAP & 11 & 84.5 & 13.51 & $65-105$ & $<0.001^{*}$ \\
E/L & 15 & 134.3 & 13.62 & $115-160$ &
\end{tabular}

*Statistically Significant Difference (P-value $<0.05)$.

Table 4. Hospital stay (days) as per management in study patients.

\begin{tabular}{cccccc}
\hline Hospital Stay (Days) & N & Mean & SD & Range & P-value \\
\hline LAP & 11 & 7.8 & 2.48 & $4-11$ & $<0.001^{\star}$ \\
E/L & 15 & 15 & 5.73 & $8-26$ & \\
\hline
\end{tabular}

${ }^{*}$ Statistically Significant Difference $(\mathrm{P}$-value $<0.05)$.

Table 5. Time of return of bowel sounds as per management in study patients

\begin{tabular}{|c|c|c|c|c|c|}
\hline \multirow{2}{*}{ Time (Days) } & \multicolumn{2}{|c|}{ LAP } & \multicolumn{2}{|c|}{$\mathrm{E} / \mathrm{L}$} & \multirow{2}{*}{ P-value } \\
\hline & No. & \%age & No. & \%age & \\
\hline Day 1 & 7 & 63.6 & 5 & 33.3 & \\
\hline Day 2 & 4 & 36.4 & 7 & 46.7 & \\
\hline Day 3 & 0 & 0.0 & 3 & 20.0 & $0.004^{*}$ \\
\hline Total & 11 & 100 & 15 & 100 & \\
\hline Mean \pm SD & \multicolumn{2}{|c|}{$1.3 \pm 0.504$} & \multicolumn{2}{|c|}{$2.1 \pm 0.743$} & \\
\hline
\end{tabular}

${ }^{*}$ Statistically Significant Difference $(\mathrm{P}$-value $<0.05)$. 
Table 6. Passage of flatus/stools as per management in study patients.

\begin{tabular}{cccccc}
\hline \multirow{2}{*}{ Time (Days) } & \multicolumn{3}{c}{ LAP } & \multicolumn{3}{c}{ E/L } & \multirow{2}{*}{ P-value } \\
\cline { 2 - 5 } & No. & \%age & No. & \%age & \\
\hline Day 2 & 5 & 45.5 & 3 & 20.0 & \\
Day 3 & 4 & 36.4 & 5 & 33.3 & \\
Day 4 & 2 & 18.2 & 6 & 40.0 & \multirow{2}{*}{$0.003^{*}$} \\
Day 5 & 0 & 0.0 & 1 & 6.7 & \\
Total & 11 & 100 & 15 & 100 & \\
Mean \pm SD & \multicolumn{2}{c}{$2.5 \pm 0.786$} & \multicolumn{2}{c}{$3.6 \pm 0.899$} & \\
\hline
\end{tabular}

${ }^{\star}$ Statistically Significant Difference $(\mathrm{P}$-value $<0.05)$.

Table 7. Complications as per management in study patients.

\begin{tabular}{cccccc}
\hline & \multicolumn{2}{c}{$\begin{array}{c}\text { Laparoscopic } \\
\text { Complications }\end{array}$} & \multicolumn{2}{c}{$\begin{array}{c}\text { Exploratory } \\
\text { Laparatomy (E/L) }\end{array}$} & \multirow{2}{*}{ P-value } \\
\cline { 2 - 4 } & No. & \%age & No. & \%age & \\
\hline Post-OperativeObstruction & 1 & 9.1 & 3 & 20.0 & 0.604 \\
Small bowel Tear in Jejunum & 1 & 9.1 & 0 & 0.0 & 1.000 \\
Post-operative ileus & 1 & 9.1 & 0 & 0.0 & 1.000 \\
Peritonitis & 0 & 0.0 & 2 & 13.3 & 0.492 \\
Anastomotic leakage & 0 & 0.0 & 3 & 20.0 & 0.238 \\
Urinary retention & 0 & 0.0 & 1 & 6.7 & 1.000 \\
Wound infection & 0 & 0.0 & 4 & 26.7 & 0.113 \\
\hline
\end{tabular}

On fallow up none of our patients developed recurrence among laparoscopic group. Only two patients in open group developed sub-acute intestinal obstruction during six month fallow up. Both the patients were managed conservatively.

\section{Discussion}

Acute small bowel obstruction is a significant cause of emergency surgical admission and morbidity. Postoperative adhesions are the most frequent cause. Even after operative intervention for small bowel obstruction, recurrences are common. Laparoscopy is believed to reduce the risk of adhesions compared to laparotomy.

Similar to our results, many studies in which laparoscopic adhesiolysis and open adhesiolysis have been compared against each other have yielded results favouring the laparoscopic method [16]-[22].

The patients in the present study were aged between 20 to 70 years with a mean age of 44.7 years. Maximum numbers of patient were between 35 - 49 years old (31.4\%). The higher mean age may be due to fact that children below 12 years of age were excluded in our study. In our study, there were 27 (52.9\%) males and $24(47.1 \%)$ female patients. The clinical spectrum of our cases was similar to that seen in Meena LN et al. (2018) [23] wherein the largest group comprised of patients between the age of 31 - 40 years and accounted for $24 \%$ of 
the cases. Only $4 \%$ of the cases were above the age of 70 years. Mean age in their study group was 40.23 years. Males were approximately thrice as frequently as females with a male to female ratio of 3.1:1. The results are also comparable to those of Liauw JJY et al. (2005) [24] who in their study found the mean age of 46.5 years (range 29 - 88 years).

In our study, there were 28 (54.9\%) patients who presented with continuous abdominal pain while as $23(45.1 \%)$ came with colicky pain. 40 (78.4\%) patients in our study had constipation. Our results are almost similar to study of Mitra A et al. (2018) [25] in which the most common symptom was found to be abdominal pain, followed by vomiting, constipation and abdominal distension.

In our study $34(66.7 \%)$ patients on admission gave history of effortless vomiting followed by projectile and sudden vomiting in $6(11.8 \%)$ patients each. A study done by Chen SC et al. [26] also found vomiting in $44 \%$ of cases which is close to our findings. Distension of abdomen was present in 29 (58\%) patients which were again found by Chen SC et al. [26] in 45.8\% patients.

In our study, adhesion obstruction was found in 27 (52.9\%) patients, malignancy in $8(15.7 \%)$ patients, fecaloma in $5(9.8 \%)$ patients, crohn's disease and tuberculosis were found in $3(5.9 \%)$ patients each, worm obstruction in 2 (3.9\%) patients while as paraduodenal hernia was found in $1(2.0 \%)$ patients. Our results are comparable with the findings of Sarr MG et al. [27] who found adhesions in $62.7 \%$, malignancy in $11.8 \%$ in their study. Miller G et al. [28] also found adhesion obstruction in $74 \%$ of their cases, Crohn's disease in $7 \%$ and neoplasia in 5\% cases. Meena LN et al. [23] alsofoundadhesions as the most frequently encountered aetiology in $39 \%$ of their patients.

On CT scan inter-loop fluid was present in $23(45.1 \%)$ patients and absent in $28(54.9 \%)$ patients. Transition point was present in $31(60.8 \%)$ patients and absent in $20(39.2 \%)$ patients. $16(31.4 \%)$ patients had $3-4 \mathrm{~cm}$ diameter of bowl while as $2-3 \mathrm{~cm}$ diameter of bowl was found in 13 (25.5\%) patients. Reddy SRR et al. [29] found that major CT criteria for small bowel obstruction include small bowel dilatation $\geq 3 \mathrm{~cm}$ in diameter without significant colonic dilatation (i.e. $<6$ $\mathrm{cm}$ colonic diameter) and a transition point from dilated to collapsed small bowel. Minor radiologic criteria for small bowel obstruction include air-fluid levels and a decompressed colon [30]. Transition point was seen in $63 \%$ of patients in their study.

There were 25 (49\%) patients in our study who were managed conservatively while as $11(21.6 \%)$ patients had undergone laparoscopic adhesiolysis and 15 (29.4\%) underwent exploratory laparotomy. Meena LN et al. [23] in their study managed 40 patients conservatively out of 70 patients. Out the 34 operated cases, 23 were performed by open method (66\%). Miller G et al. [28] also had similar observation, their $70 \%$ patients were managed conservatively.

An average operative time for laparoscopic adhesiolysis was 84.5 minutes and explorative laparotomy was 134.3 minutes. The difference was statistically significant. Otani $\mathrm{K}$ et al. [31] showed that the operative time of laparoscopic sur- 
gery tended to be shorter than that of open surgery (96.0 minutes versus 53.0 minutes). Lujan $\mathrm{HJ}$ et al. [32] foundmean operative time for laparoscopic group was 59 minutes while as it was 97 minutes in conventional open adhesiolysis. Mean operative time in minute was again confirmed by Liauw JJY et al. [24] where the mean operating time in laparoscopic group was 74 minutes.

Patients who underwent laparoscopic adhesiolysiss stay postoperatively in hospitals for 7.8 days while as patients who underwent explorative laparotomy were discharged after an average of 15 days. This was statistically significant. Our results are somewhat similar to that of Miller G et al. [28] who in their study found the mean hospital stay (in days) of 12 days after explorative laparatomy. Another study done by Brolein RE et al. [33] reported that mean length of hospital stay in conservative group and operated group was 6.9 and 7.7 days respectively. Average post-operative stay in a study done by Mitra A et al. [25] was 4.61 days in laparoscopy group and 12.91 days in open group. Wellstein [19] reported duration of hospital stay at 11.3 days for laparoscopy group.

In our study mean time (days) for passage of flatus/stools after surgery was 2.5 \pm 0.786 days in laparoscopic adhesiolysiss and $3.6 \pm 0.899$ days in exploratory laparotomy. Khaikin et al. [34] observed a significant reduction time to first bowel movement in patients treated with laparoscopy while Wullstein et al. [19] showed 3.5 days as the recovery of postoperative bowel movements in laparoscopic group and 4.4 days in open adhesiolysis group. We were able to show almost same results that could be found in literature. In our study mean time (days) for return of bowel sounds was $1.3 \pm 0.504$ days in laparoscopic adhesiolysiss and $2.1 \pm 0.743$ days in exploratory laparotomy which was statistically significant. There were complications in both operative groups; however, the number was insufficient to comment upon statistically.

Postoperative obstruction was seen in 1 (9.1\%) patients of laparoscopic adhesiolysis while as $3(20 \%)$ patients developed postoperative obstruction in exploratory laparotomy group. Anastomotic leakage was seen again in $3(20.0 \%)$ patients of exploratory laparotomy. Small bowel tear in jejunum, postoperative ileus were found in 1 (9.1\%) each in laparoscopic adhesiolysiss group. Wound infection was found in $4(26.7 \%)$ patients in exploratory laparotomy group while as there was no patients with wound infection in laparoscopic adhesiolysis group. Wullstein C et al. (2003) [19] in their study found postoperative wound infection in 3 patients in laparoscopic group and 6 patients in open adhesiolysiss. They also observed anastomotic leakage in 2 patients in open adhesiolysiss and none in laparoscopic group. Navez et al. (1996) [35] in their study observed bowel injury in $9 \%$ patients in laparoscopic adhesiolysis, Ibrahim et al. (1996) [36] also found bowel injury in $9.1 \%$ of patients in laparoscopic adhesiolysis. Byrne J et al. (2014) [20] observed urinary tract infection in 3.8\% and $1.2 \%$ and prolonged ileus ( $>5$ days) in $6.8 \%$ and $4.0 \%$ in open and laparoscopic groups respectively. Anastomotic leak in their study was found in $3.2 \%$ patients in open adhesiolysis and none in laparoscopic adhesiolysis. 
There was no mortality in laparoscopic adhesiolysiss group while as only 1 (6.7\%) patient died in exploratory laparotomy group. The 30-day mortality rate of $4.7 \%$ in the open adhesiolysis group compared with $1.3 \%$ in the laparoscopic adhesiolysisgroup was observed by Kelly KN et al., (2014) [22].

\section{Conclusion}

Small bowel obstruction (SBO) is a common reason for admission in our emergency department. Laparoscopy is safe and effective tool for the management of acute intestinal obstruction provided performed by skilled surgeons and in selected patients. Laparoscopic adhesiolysis for small bowel obstruction has number of potential advantages like less operative pain, quicker return of intestinal function, shorter hospital stay, reduced recovery time, fewer wound complications and decreased postoperative adhesion formation. However, despite the fact that it is so commonly used and technically advanced, SBO is still a condition where the use of laparoscopy is limited in everyday practice mainly to selected cases such as adhesive SBO caused by single adhesions or foreign bodies in the gastrointestinal tract. The limitation of laparoscopy is its use in complicated SBO and therefore high conversion rate.

\section{Conflicts of Interest}

The author declares no conflicts of interest regarding the publication of this paper.

\section{References}

[1] Houghton, S.G., Del Medina, A.R. and Sarr, M.G. (2007) Bowel Obstruction. In: Zinner, M.J. and Ashley, S.W., Eds., Maingot's Abdominal Operations, 11th Edition, McGraw-Hill, New York, 479.

[2] Mullan, C.P., Siewert, B. and Eisenberg, R.L. (2012) Small Bowel Obstruction. AJR, 198, 105-116. https://doi.org/10.2214/AJR.10.4998

[3] Dite, P., Lata, J. and Novotny, I. (2003) Intestinal Obstruction and PerforationThe Role of Gastroenterologist. Digestive Diseases, 21, 63-67. https://doi.org/10.1159/000071341

[4] Landercasper, J., Cogbill, T.H., Merry, W.H., et al. (1993) Long Term Outcome after Hospitalization for Small Bowel Obstruction. The Archives of Surgery, 128, 765-770. https://doi.org/10.1001/archsurg.1993.01420190059008

[5] Mucha, P. (1987) Small Intestinal Obstruction. Surgical Clinics of North America, 67, 597-620.

[6] Maglinte, D.D.T., Balthazar, E.J., Kelvin, F.M., et al. (1997) The Role of Radiology in the Diagnosis of Small Bowel Obstruction. AJR, 168, 1171-1180. https://doi.org/10.2214/ajr.168.5.9129407

[7] Khanzada, T.W., Samad, A. and Champa, S. (2007) Aetiologic Spectrum of Dynamic Intestinal Obstruction. Gomal Journal of Medical Sciences, 5, 59.

[8] Wasadikar, P.P. and Kulkarni, A.B. (1997) Intestinal Obstruction Due to Ascariasis. British Journal of Surgery, 84, 410-412. https://doi.org/10.1002/bjs.1800840345

[9] Cappel, M.S. and Batke, M. (2008) Mechanical Obstruction of the Small Bowel and 
Colon. Medical Clinics of North America, 92, 575-597. https://doi.org/10.1016/j.mcna.2008.01.003

[10] Maglinte, D.D., Heitkamp, D.E. and Howard, T.J. (2003) Current Concepts in Imaging of Small Bowel Obstruction. Radiologic Clinics of North America, 41, 263-283.

[11] Bass, K.N., Jones, B. and Bulbley, G.B. (1997) Current Management of Small Bowel Obstruction. Advances in Surgery, 31, 1-34.

[12] Barkan, H., Webster, S. and Ozeran, S. (1995) Factors Predicting the Recurrence of Adhesive Small Bowel Obstruction. Annals of Surgery, 170, 361-365.

[13] O'Connor, D.B. and Desmond, C. (2012) The Role of Laparoscopy in the Management of Acute Small Bowel Obstruction: A Review of over 2000 Cases. Surgical Endoscopy, 26, 12-17.

[14] Nagle, A., Ujiki, M., Denham, W. and Murayama, K. (2004) Laparoscopic Adhesiolysis for Small Bowel Obstruction. The American Journal of Surgery, 187, 464-470. https://doi.org/10.1016/j.amjsurg.2003.12.036

[15] Wullstein, C. and Gross, E. (2003) Laparoscopic Compared with Conventional Treatment of Acute Adhesive Small Bowel Obstruction. British Journal of Surgery, 90, 117-1151. https://doi.org/10.1002/bjs.4177

[16] Chopra, R., McVay, C., Phillips, E., et al. (2003) Laparoscopic Lysis of Adhesions. The American Surgeon, 69, 966-968.

[17] Davies, S.W., Gillen, J.R., Guidry, C.A., et al. (2014) A Comparative Analysis between Laparoscopic and Open Adhesiolysis at a Tertiary Care Center. The American Surgeon, 80, 261-269.

[18] Khaikin, M., Schneidereit, N., Cera, S., et al. (2007) Laparoscopic vs. Open Surgery for Acute Adhesive Small-Bowel Obstruction: Patients' Outcome and Cost-Effectiveness. Surgical Endoscopy, 21, 742-746. https://doi.org/10.1007/s00464-007-9212-1

[19] Wullstein, C. and Gross, E. (2003) Laparoscopic Compared with Conventional Treatment of Acute Adhesive Small Bowel Obstruction. British Journal of Surgery, 90, 1147-1151.

[20] Byrne, J., Saleh, F., Ambrosini, L., et al. (2015) Laparoscopic versus Open Surgical Management of Adhesive Small Bowel Obstruction: A Comparison of Outcomes. Surgical Endoscopy, 29, 2525-2532.

[21] Saleh, F., Ambrosini, L., Jackson, T., et al. (2014) Laparoscopic versus Open Surgical Management of Small Bowel Obstruction: An Analysis of Short-Term Outcomes. Surgical Endoscopy, 28, 2381-2386.

[22] Kelly, K.N., Iannuzzi, J.C., Rickles, A.S., et al. (2014) Laparotomy for Small Bowel Obstruction: First Choice or Last Resort for Adhesiolysis? A Laparoscopic Approach for Small-Bowel Obstruction Reduces 30-Day Complications. Surgical Endoscopy, 28, 65-73.

[23] Meena, L.N., Bhatt, N. and Jain, S. (2018) Small Bowel Obstruction: Need for Surgery Based on History and Radiology. International Surgery Journal, 5, 588-592.

[24] Liauw, J.J.Y. and Cheah, W.K. (2005) Laparoscopic Management of Acute Small Bowel Obstruction. Asian Journal of Surgery, 28, 185-188.

[25] Mitra, A., Pandya, D. and Chandak, U. (2018) Study of Laparoscopic Adhesiolysis in Postoperative Adhesive Intestinal Obstruction. Radiology, 3, 96-101.

[26] Chen, S.C., Chang, K.J., Lee, P.H., Wang, S.M., Chen, K.M. and Lin, F.Y. (1999) Oral Urografin in Postoperative Small Bowel Obstruction. World Journal of Sur- 
gery, 23, 1051-1054. https://doi.org/10.1007/s002689900622

[27] Sarr, M.G., Bulkley, G.B. and Zuidema, G.D. (1983) Preoperative Recognition of Intestinal Strangulation Obstruction: Prospective Evaluation of Diagnostic Capacity. The American Journal of Surgery, 145, 176. https://doi.org/10.1016/0002-9610(83)90186-1

[28] Miller, G., Boman, J., Shrier, I. and Gordon, P.H. (2000) Etiology of Small Bowel Obstruction. The American Journal of Surgery, 180, 33-36.

[29] Reddy, S.R.R. and Cappell, M.S. (2017) A Systematic Review of the Clinical Presentation, Diagnosis, and Treatment of Small Bowel Obstruction. Current Gastroenterology Reports, 19, 28. https://doi.org/10.1007/s11894-017-0566-9

[30] Paulson, E.K. and Thompson, W.M. (2015) Review of Small Bowel Obstruction: The Diagnosis and When to Worry. Radiology, 275, 332-342. https://doi.org/10.1148/radiol.15131519

[31] Otani, K., Ishihara, S., Nozawa, H., Kawai, K., Hata, K., Kiyomatsu, T., et al. (2017) A retrospective Study of Laparoscopic Surgery for Small Bowel Obstruction. Annals of Medicine and Surgery, 16, 34-39. https://doi.org/10.1016/j.amsu.2017.02.045

[32] Lujan, H.J., Oren, A., Plasencia, G., Canelon, G., Gomez, E., Hernandez-Cano, A. and Jacobs, M. (2006) Laparoscopic Management as the Initial Treatment of Acute Small Bowel Obstruction. Journal of the Society of Laparoendoscopic Surgeons, 104, 466-472.

[33] Brolein, R.E. (1984) Partial Small Bowel Obstruction. Surgery, 95, 145-149.

[34] Johnson, K.N., et al. (2012) Laparoscopic Management of Acute Small Bowel Obstruction: Evaluating the Need for Resection. Journal of Trauma and Acute Care Surgery, 72, 25-30. https://doi.org/10.1097/TA.0b013e31823d8365

[35] Navez, B., Arimont, J.M. and Guit, P. (1998) Laparoscopic Approach in Acute Small Bowel Obstruction: A Review of 68 Patients. Hepato-Gastroenterology, 45, 21462150.

[36] Ibrahim, I.M., Wolodiger, F., Sussman, B., et al. (1996) Laparoscopic Management of Acute Small Bowel Obstruction. Surgical Endoscopy, 10, 1012-1015. https://doi.org/10.1007/s004649900226 